\section{INDEX TO ADVERTISEMENTS}

Abbott Laboratories, Depakane - (iv) and (v)

DISA - (vii)

Geigy, Tegretol - (x), (xi) and (xvi)

Geigy, Lioresal — outside back cover, (viii) and (xii)

Grass Instruments,

Polysomnographic Recording - (iii)

Heerbrugg Microscopes - (ix)

Parke Davis, Dilantin — inside back cover and (xviii)

Sandoz, Fiorinal - inside front cover and (xiv)

Sandoz, Sandomigran - (xiv) and (xv)

Unimed Canada, Serc - (xiii)

\section{NEUROLOGIST}

The Sir Mortimer B. Davis-Jewish General Hospital, a 600 bed McGill University Teaching Hospital currently has a geographic full time (GFT) position available for a neurologist. The Hospital is part of the McGill Teaching Program in Neurology and the successful candidate will receive an appointment as an Assistant Professor in the Department of Neurology and Neurosurgery at McGill University.

Interested candidates should direct their inquiries and curriculum vitae to:

Israel Libman, M.D., F.R.C.P.(C)

Chief, department of Neurology

5750 Côte des Neiges, Suite \#208

Montreal, Quebec,

H3S 1 Z8

\section{BRIEF PRESCRIBING INFORMATION}

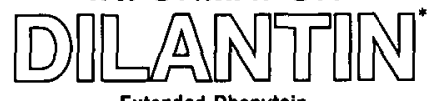

Extended Phenytoin

Sodium Capsules, U.S.P. $100 \mathrm{mg}$

ANTICONVULSANT

INDICATIONS

Dilantin is indicated for the control of generalized tonic-clonic (grand mal) seizures and complex partial (psychomotor) seizures.

\section{CONTRAINDICATIONS}

Dilantin is contraindicated in those patients with a history of hypersensitivity to hydantoin products.

\section{WARNINGS}

Abrupt withdrawal of phenytoin in epileptic patients may precipitate status epilepticus. Phenytoin is not indicated in seizures due to hypoglycemia or other causes which may be immediately identified and corrected.

Phenytoin metabolism may be significantly altered by the concomitant use of other drugs such as:

A Barbiturates may enhance the rate of metabolism of phenytoin. This effect, however, is variable and unpredictable. It has been reported that in some patients the concomitant administration of carbamazepine resulted in an increased rate of phenytoin metabolism.

B Coumarin anticoagulants, disulfiram, phenylbutazone, and sulfaphenazole may in hibit the metabolism of phenytoin, resulting in increased serum levels of the drug. This may lead to an increased incidence of nystagmus, ataxia, or other toxic signs.

C Isoniazid inhibits the metabolism of phenytoin so that with combined therapy, patients who are slow acetylators may suffer from

\section{phenytoin intoxication}

D Tricyclic antidepressants in high doses may precipitate seizures, and the dosage of phenytoin may have to be adjusted accor. dingly.

Usage in Pregnancy: The effects of Dilantin in human pregnancy and nursing infants are unknown.

The prescribing physician will have to determine the risk/benefit in treating or counselling epileptic women of childbearing potential.

PRECAUTIONS

The liver is the chief site of biotransformation of phenytoin, patients with impaired liver function may show early signs of toxicity. Elderly patients or those who are gravely ill may show early signs of toxicity.

A small percentage of individuals who have been treated with phenytoin have been shown to metabolize the drug slowly. Slow metabolism may be due to limited enzyme availability and lack of induction; it appears to be genetically determined.

Phenytoin has been associated with reversible lymph node hyperplasia. If lymph node enlargement occurs in patients on phenytoin, every effort should be made to substitute another anticonvulsant drug or drug combination.

Drugs that control generalized tonic-clonic (grand mal) seizures are not effective for absence (petit mal) seizures. Therefore, if both conditions are present, combined drug therapy is needed.

Hyperglycemia, resulting from the drug's inhibitory effect on insulin release, has been reported. Phenytoin may also raise the blood sugar level in persons already suffering from hyperglycemia.

\section{ADVERSE REACTIONS}

Central Nervous System: The most common manifestations encountered with phenytoin therapy include nystagmus, ataxia, slurred speech, and mental confusion. Dizziness, insomnia, transient nervousness, motor twitchings, and headache have also been observed. These side effects may disappear with continuing therapy at a reduced dosage level.

Gastrointestinal System: Phenytoin may cause nausea, vomiting, and constipation. Administration of the drug with or immediately after meals may help prevent gastro. intestinal discomfort.

Integumentary System: Dermatological manifestations sometimes accompanied by fever have included scarlatiniform or morbilliform rashes.

Hemopoietic System: Hemopoietic complications, some fatal, have occasionally been reported in association with administration of phenytoin. These have included thrombocytopenia, leukopenia, granulocytopenia, agranulocytosis, and pancytopenia.

Other: Gingival hyperplasia occurs frequently; this incidence may be reduced by good oral hygiene including gum massage, frequent brushing and appropriate dental care. Polyarthropathy and hirsutism occur occasionally. Hyperglycemia has been reported. Toxic hepatitis, liver damage, and periarteritis nodosa may occur and can be fatal.

MANAGEMENT OF OVERDOSAGE

The mean lethal dose in adults is estimated to be 2 to 5 grams. The cardinal initia symptoms are hystagmus, ataxia and dysarthria. The patient then becomes comatose, the pupils are unresponsive and hypotension occurs. Death is due to respiratory depression and apnea. Treatment is non specific since there is no known antidote. First, the stomach should be emptied. If the gag reflex is absent, the airway should be supported. Oxygen, vasopressors and assisted ventilation may be necessary for central nervous system, respiratory and cardiovascular depression. Finally, hemodialysis can be considered since phenytoin is not completely bound to plasma proteins. DOSAGE AND ADMINISTRATION

Dosage should be individualized to provide maximum benefit. In some cases, serum blood level determinations may be necessary for optimal dosage adjustments - the clinically effective serum level is usually $10-20 \mathrm{mcg} / \mathrm{mL}$.

Adult Dose: Patients who have received no previous treatment may be started on one $100 \mathrm{mg}$ Dilantin Capsule three times daily and the dose then adjusted to suit individual requirements.

Pediatric Dose: Initially, $5 \mathrm{mg} / \mathrm{kg} /$ day in two or three equally divided doses, with subsequent dosage individualized to a maximum of $300 \mathrm{mg}$ daily. A recommended daily maintenance dosage is usually 4 to $8 \mathrm{mg} / \mathrm{kg}$. Children over 6 years old may require the minimum adult dose $(300 \mathrm{mg} /$ day). Pediatric dosage forms available include a $30 \mathrm{mg}$ Capsule, a $50 \mathrm{mg}$ palatably flavoured Infatab or an oral suspension form containing $30 \mathrm{mg}$ of Dilantin in each $5 \mathrm{~mL}$.

Alternative Dose: Once-a-day dosage for adults with $300 \mathrm{mg}$ of Dilantin may be considered if seizure control is established with divided doses of three $100 \mathrm{mg}$ Capsules daily.

HOW SUPPLIED

Dilantin $100 \mathrm{mg}$ Capsules; in bottles of $100 \& 1000$.

Complete prescribing information available upon request.

PARKE-DAVIS

Parke-Davis Canada Inc. Scarborough, Ontario

*Reg. T.M. Parke Davis \& Company Parke-Davis Canada inc., auth. use 


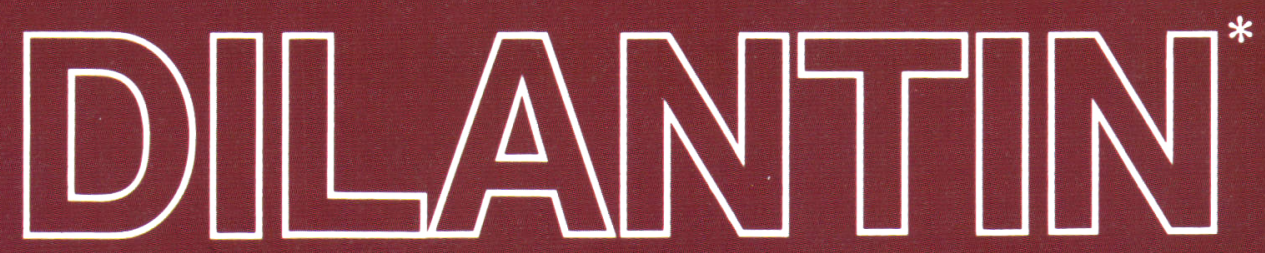

\section{Extended Phenytoin Sodium Capsules U.S.P. A RECOCNIZED DIFFERENCE}

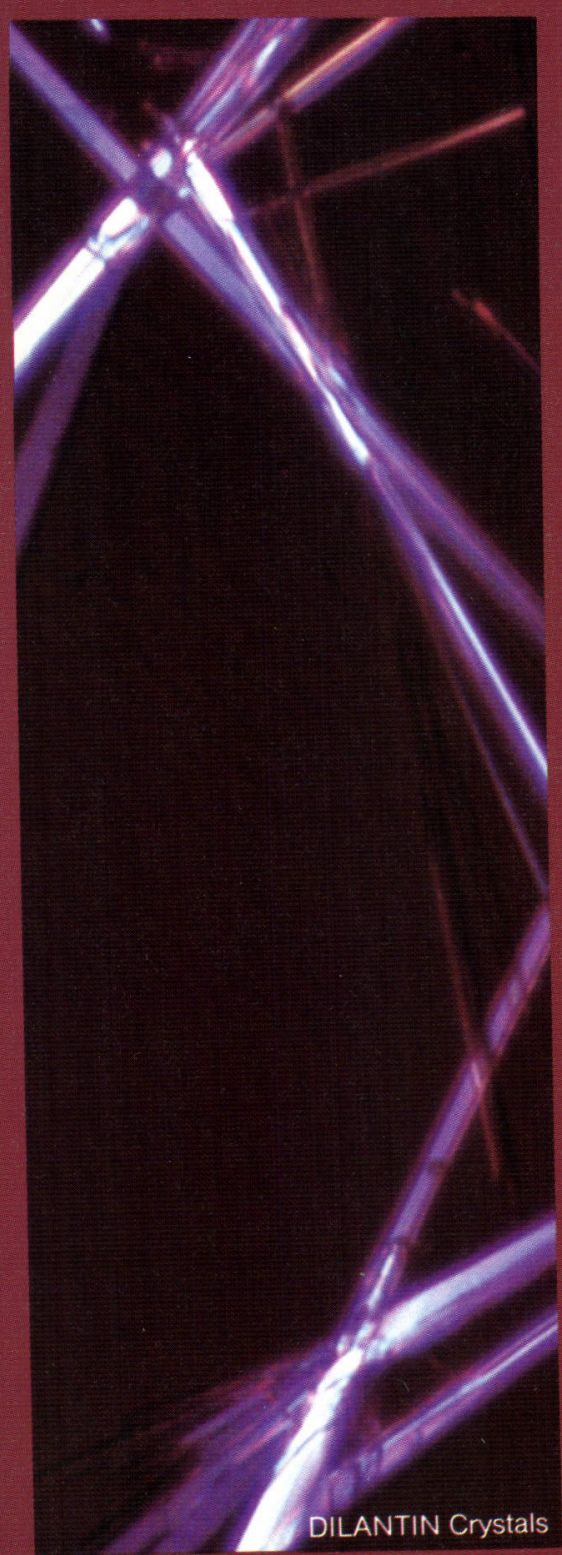

\section{USP XX now differentiates between Extended and Prompt Phenytoin Sodium Capsules.}

Extended phenytoin sodium has been recognized as a distinct pharmaceutical entity. Its slow dissolution and absorption do not create significant fluctuations in phenytoin blood levels.
Prompt phenytoin sodium has a faster dissolution and higher initial blood levels. The two forms of phenytoin sodium are not interchangeable.**

\section{DILANTIN Capsules have not changed.}

Extended effect has always been the action of DILANTIN therapy. Only the U.S.P. standards have changed to recognize the difference between "extended" and "prompt" phenytoin sodium. Both you and your patient can continue to benefit from the consistent antiepileptic action of DILANTIN capsules.

\section{Once-daily-dosage option is confirmed for DILANTIN Capsules.}

Extended action of DILANTIN offers greater convenience and improved patient compliance. Dependable, effective therapy is now available through a oncedaily-dosage option, once seizure control has been established with divided doses.

\section{DILANTIN formulation ensures dependable bioavaliability.}

Extended phenytoin classification of DILANTIN capsules is the result of its unique dissolution profile. Due to its special formulation, DILANTIN exerts a slow, steady release of phenytoin for dependable bioavailability.

**Patients should be maintained on one form of phenytoin (extended or prompt) to avoid toxicity or loss of seizure control.

\section{START WITH DILANTIN-STAY WITH DILANTIN FOR OVER A GENERATION, THE STANDARD IN EPILEPSY MANAGEMENT}


